\title{
Emergence and decay rate of the edge plasma flow shear near a critical transition
}

\author{
J M Delgado ${ }^{1}$, L Garcia ${ }^{1}$ and B A Carreras ${ }^{2}$ \\ ${ }^{1}$ Universidad Carlos III, 28911 Leganés, Madrid, Spain \\ ${ }^{2}$ BACV Solutions, Inc, Oak Ridge, Tennessee 37830, USA \\ E-mail: jmdelgad@fis.uc3m.es
}

\begin{abstract}
Recently, the experimental results for the emergence of the plasma shear flow layer in TJ-II have been explained as a second-order phase transition like process by using a simple model of envelope equations for the fluctuation level, the averaged poloidal velocity shear and the pressure gradient (2006 Phys. Plasmas 13 122509). Here, we extend this model by incorporating radial coupling. The model is applied to the study of the turbulence-shear flow interaction when the energy flux is low. Transition dynamics and their concomitant thresholds are examined within the context of this model. The effect of an external torque induced by electrode biasing has also been considered. In particular, we analyze the decay rate of the shear flow after switching off the biasing.
\end{abstract}

\section{Introduction}

The importance of shear flows in magnetically confined plasmas is widely recognized. This is in part, due to the role that these flows play in improving confinement by turbulence suppression [1,2]. A current problem in plasma physics is to understand how shear flows are created and how they interact with turbulence. This is a complex problem that can be approached at different levels, going from direct numerical simulations of the plasma to the development of simplified reduced models. In this paper we follow the latter approach.

The spontaneous formation of shear flows with nontrivial radial structure is studied. Due to the lack of sufficiently high spatial and temporal resolution, it is difficult to formulate an experimental test that will definitively select the dominant mechanisms responsible for the transition. However recent TJ-II [3] experimental results offer the possibility of improved diagnostics with increased radial resolution during and after the transition [4-6]. Motivated by this, we investigate in this paper the radial structure of the turbulence and poloidal flow in 
the context of phase transition models based on the one initially proposed [7] to explain the transition from the low confinement mode to the high confinement mode [8].

For low input power, there is a transition to a regime in which the fluctuation level decreases or increases at a slower rate than the input flux, and shear flow is spontaneously developed. We focus our attention on this transition in our starting model.

The model we use for our study is a modified version of a fluctuation-flow model with radial structure [9]. The fluctuation-flow model consists of three coupled partial differential equations of the reaction-diffusion type with nonlinear diffusivities for the averaged poloidal velocity shear, the envelope of the turbulence fluctuations level, and the pressure. We include the dependence of the coefficients on the pressure gradient [10]. We have derived an expression for the Reynolds stress term in the averaged poloidal flow equation which conserves angular momentum.

The effect of electrode biasing is included in the model through an external torque term in the poloidal velocity shear equation. The relaxation of flows is studied by applying the electrode biasing during a time, and then switching off the biasing to analyze the decay. The decay is algebraic when we are close to the critical energy flux, and the decay time tends to infinity as the flux tends to the critical value.

The rest of this paper is organized as follows. The transport model and the derivation of the Reynolds stress term are presented in section 2 . The main results of the model are shown in section 3. The effect of electrode biasing and its relation to experimental measurements are discussed in section 4. Finally, the conclusions of this paper are presented in section 5.

\section{Transport model}

The model is formulated in terms of three fields: the averaged turbulence fluctuation level $\mathcal{E} \equiv\left\langle\left(\tilde{n} / n_{0}\right)^{2}\right\rangle^{1 / 2}$, where $n_{0}$ is the equilibrium plasma density and $\tilde{n}$ is the fluctuation density; the poloidal flow shear, $V^{\prime} \equiv \partial\left\langle V_{\theta}\right\rangle / \partial r$, where $\left\langle V_{\theta}\right\rangle$ denotes poloidal flow averaged over a magnetic flux surface and the flux-surface averaged pressure, $\mathcal{P}$.

$$
\begin{aligned}
\frac{\partial \mathcal{E}}{\partial \bar{t}} & =\gamma \mathcal{E}-\alpha_{1} \mathcal{E}^{2}-\alpha_{2} V^{\prime 2} \mathcal{E}+\frac{1}{r} \frac{\partial}{\partial r}\left[r \mathcal{D}(r) \frac{\partial \mathcal{E}}{\partial r}\right], \\
\frac{\partial V^{\prime}}{\partial \bar{t}} & =-\mu V^{\prime}-\frac{\partial}{\partial r}\left[\frac{1}{r^{2}} \frac{\partial}{\partial r}\left(r^{2}\left\langle\tilde{V}_{r} \tilde{V}_{\theta}\right\rangle\right)\right], \\
\frac{\partial \mathcal{P}}{\partial \bar{t}} & =\mathcal{S}+\frac{1}{r} \frac{\partial}{\partial r}\left[r \mathcal{D}(r) \frac{\partial \mathcal{P}}{\partial r}\right] .
\end{aligned}
$$

This is a one-dimensional model in which quantities are assumed to depend only on the radial coordinate, $r$. The first term in the equation for $\mathcal{E}$ represents a pressure driven, linear growth of the fluctuations. The second term on the right-hand side models the saturation of turbulence in the absence of shear flow and the third term models the turbulence suppression by shear flow. Here we assume low energy flux so we can neglect diamagnetic effects. The first term on the right-hand side of equation (2) models the poloidal flow shear damping due to magnetic pumping, while the second term is the contribution from the Reynolds stress.

Because of the bad magnetic field line curvature at the stellarator edge, we assume that the basic instability underlying the turbulence at the edge of TJ-II is the resistive interchange mode. Assuming mixing length saturation of the turbulence, we have

$$
\alpha_{1}=\frac{1}{\Lambda^{7 / 6}} \frac{\gamma}{W_{k}}\left(-\frac{1}{\mathcal{P}} \frac{\mathrm{d} \mathcal{P}}{\mathrm{d} r}\right)^{-1}, \quad \alpha_{2}=\frac{k_{\theta}^{2} W_{k}^{2}}{\gamma},
$$

where $W_{k}$ is the mode width and $\Lambda$ is the enhancement factor at saturation [11]. 
Since we are only interested in the shear layer region at the plasma edge, a slab approximation is appropriate. Setting $\bar{x}=r-r_{\mathrm{s}}$, where $r_{\mathrm{s}}$ is the radial position of the left end of the shear layer region, $\partial / \partial r \rightarrow \partial / \partial \bar{x}$ in equations (1)-(3), and the different $r$-factors can be approximated as constants. In order to get a dimensionless set of equations, we define the dimensionless variables $t$ and $x$ as $\mu \bar{t}$ and $\bar{x} / L$, respectively. Here, $L$ is the length of the shear layer region, so $x$ goes from 0 to 1 . For the three fields, we change to the variables $E=\mathcal{E} / \mathcal{E}_{0}$, $\sigma=V^{\prime} / V_{0}^{\prime}$ and $p=\mathcal{P} / \mathcal{P}_{0}$, where the constants $\mathcal{E}_{0}, V_{0}^{\prime}$ and $\mathcal{P}_{0}$ are going to be defined shortly.

From the dependence of the coefficients on the linear growth rate, $\gamma$, and mode width, $W_{k}$, we can derive the dependence of the coefficients on the pressure gradient. We assume that all other dependences are weak and we take the coefficients to depend only on $N \equiv|\partial p / \partial x|$. Since $\gamma=\gamma_{0} N^{2 / 3}$ and $W_{k}=W_{0} N^{1 / 6}[11], \alpha_{1}=\bar{\alpha}_{1} N^{-1 / 2}$ and $\alpha_{2}=\bar{\alpha}_{2} N^{-1 / 3}$, where

$$
\bar{\alpha}_{1}=\frac{\gamma_{0}}{\Lambda^{7 / 6}} \frac{L}{W_{0}} \frac{\mathcal{P}}{\mathcal{P}_{0}}, \quad \bar{\alpha}_{2}=\frac{k_{\theta}^{2} W_{0}^{2}}{\gamma_{0}} .
$$

By choosing

$$
\frac{\mathcal{P}_{0}}{L}=-\left(\frac{\mu}{\gamma}\right)^{3 / 2} \frac{\mathrm{d} \mathcal{P}}{\mathrm{d} r}, \quad \mathcal{E}_{0}=\Lambda^{7 / 6} \frac{W_{0}}{L} \frac{\mathcal{P}_{0}}{\mathcal{P}}, \quad V_{0}^{\prime}=\frac{\mu}{k_{\theta} W_{0}},
$$

we eliminate the explicit dependence on the parameters $\gamma_{0}, \bar{\alpha}_{1}$ and $\bar{\alpha}_{2}$, and the dimensionless set of equations is

$$
\begin{aligned}
& \frac{\partial E}{\partial t}=N^{2 / 3} E-N^{-1 / 2} E^{2}-N^{-1 / 3} \sigma^{2} E+\frac{\partial}{\partial x}\left[\left(D_{1} E+D_{0}\right) \frac{\partial E}{\partial x}\right], \\
& \frac{\partial \sigma}{\partial t}=-\sigma-\alpha_{3} \frac{\partial^{2}}{\partial x^{2}}\left(N^{-4 / 3} E^{2} \sigma\right)-\frac{\partial^{2}}{\partial x^{2}}\left[\left(D_{2} N^{-5 / 3} E^{2}+D_{3}\right) \frac{\partial^{2} \sigma}{\partial x^{2}}\right], \\
& \frac{\partial p}{\partial t}=S+\frac{\partial}{\partial x}\left[\left(D_{1} E+D_{0}\right) \frac{\partial p}{\partial x}\right] .
\end{aligned}
$$

The second and third terms on the right-hand side of equation (8) come from the Reynolds stress. The expression for Reynolds stress contributions is derived in appendix A. The quasilinear calculation is similar to the one proposed in [12] and has two terms. The one responsible for the generation of flow is a negative viscosity term and the other is a hyper-viscosity term and damps the poloidal flow. The Reynolds stress contributions to the poloidal shear flow equation conserve angular momentum. From expression (A.7), we get

$$
\alpha_{3}=\frac{1}{2}\left(\frac{\mathcal{P}}{\mathcal{P}_{0}}\right)^{2} \mathcal{E}_{0}^{2}=\frac{1}{2} \Lambda^{7 / 3}\left(\frac{W_{0}}{L}\right)^{2} .
$$

The terms involving spatial derivatives are diffusion terms. In equations (7) and (9) these terms have the standard Fick's Law structure, with $D_{0}$ representing the collisional diffusion and $D_{1} E$ representing a renormalized turbulent diffusion. The structure of the negative diffusion operator in equation (8) is due to the fact that is an equation for the poloidal flow shear, the derivative of the poloidal flow.

We assume that the energy source term $S(x)$ is zero in this layer and that the system is driven by an energy flux $\Gamma_{0}$ from the core, which determines the boundary condition at $x=0$ according to

$$
\Gamma_{0}=-\left.\left(D_{1} E+D_{0}\right) \frac{\partial p}{\partial x}\right|_{0}, \quad p(1)=0
$$


For the other equations we use zero derivative boundary conditions:

$$
\begin{aligned}
& \left.\frac{\partial E}{\partial x}\right|_{0}=\left.\frac{\partial E}{\partial x}\right|_{1}=0, \\
& \left.\frac{\partial \sigma}{\partial x}\right|_{0}=\left.\frac{\partial \sigma}{\partial x}\right|_{1}=0 .
\end{aligned}
$$

Since equation (8) involves fourth-order derivatives in $\sigma$, we need to add another boundary condition for $\sigma$. We take

$$
\left.\frac{\partial^{3} \sigma}{\partial x^{3}}\right|_{0}=\left.\frac{\partial^{3} \sigma}{\partial x^{3}}\right|_{1}=0
$$

\section{Analytic and numerical solutions}

\subsection{Stationary, homogeneous states}

For stationary solutions, equation (9) is readily integrated, giving a relation between $E(x)$ and $N(x)$,

$$
\left(D_{1} E+D_{0}\right) N=\Gamma_{0} .
$$

Apart from the trivial solution, $E=\sigma=0$, equations (7) and (8) have one fixed point solution with $\sigma=0, E=E_{0}$ and $N=N_{0}$. From equation (7),

$$
E_{0}=N_{0}^{7 / 6} \text {. }
$$

By substitution of equation (16) in relation (15), we obtain the (constant) density gradient for the fixed point solution,

$$
D_{1} N_{0}^{13 / 6}+D_{0} N_{0}-\Gamma_{0}=0
$$

\subsection{Stability of solutions near the fixed point}

For solutions close to the fixed point is possible to derive a simplified description of the system using a multiple scale perturbation analysis. As a first step, we introduce a small parameter $\delta$ representing the size of the perturbation, and consider the following expansion:

$$
\sigma=\delta \sigma_{1}, \quad E=E_{0}+\delta^{2} E_{2}, \quad N=N_{0}+\delta^{2} N_{2}
$$

By substituting the expansion in equation (8), and taking into account equation (16), we get at first order

$$
\frac{\partial \sigma_{1}}{\partial t}=-\sigma_{1}-\alpha_{3} N_{0} \frac{\partial^{2} \sigma_{1}}{\partial x^{2}}-\left(D_{2} N_{0}^{2 / 3}+D_{3}\right) \frac{\partial^{4} \sigma_{1}}{\partial x^{4}} .
$$

We try as solutions modes like $\sigma_{1}=\sigma_{10} \cos (k \pi x)$ which satisfy the boundary conditions. To study their stability properties, we consider a temporal and spatial dependence like

$$
\sigma(x, t)=\delta \sigma_{10} e^{\gamma t} \cos (k \pi x) .
$$

Then, we get from equation (19),

$$
\gamma=-1+\alpha_{3} N_{0}(k \pi)^{2}-\left(D_{2} N_{0}^{2 / 3}+D_{3}\right)(k \pi)^{4} .
$$

This means that the range of possible unstable modes is given by the relation

$$
k_{-}<k<k_{+} \text {, }
$$




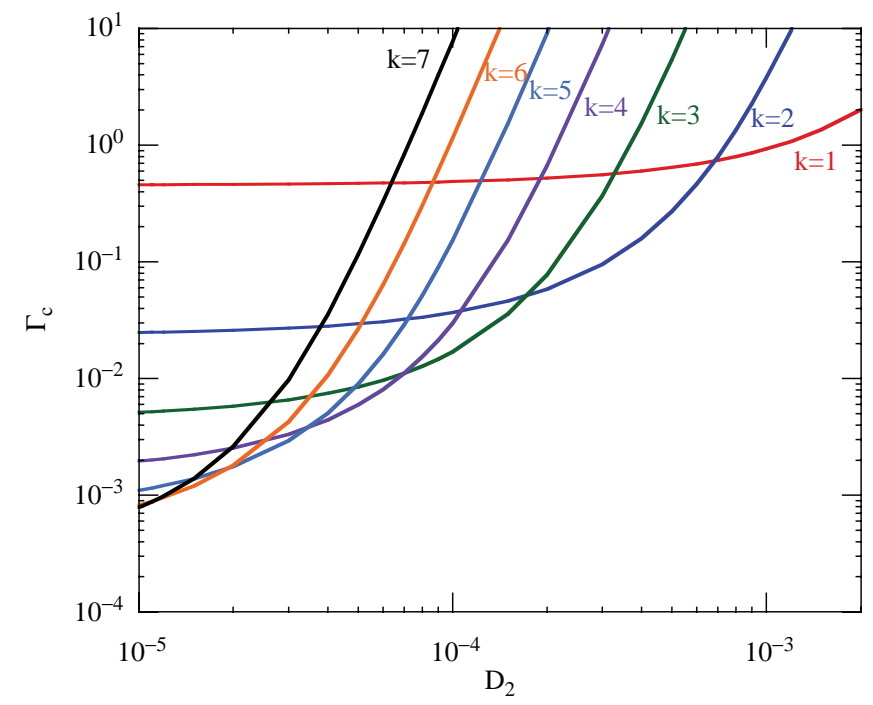

Figure 1. Flux threshold $\Gamma_{\mathrm{c}}$ as a function of the hyperdiffusivity coefficient $D_{2}$ for various values of the wavenumber $k$. The rest of parameters are given in the text.

where

$$
\left(k_{ \pm} \pi\right)^{2}=\frac{\alpha_{3} N_{0} \pm \sqrt{\left(\alpha_{3} N_{0}\right)^{2}-4\left(D_{2} N_{0}^{2 / 3}+D_{3}\right)}}{2\left(D_{2} N_{0}^{2 / 3}+D_{3}\right)} .
$$

From this expression, we obtain a necessary condition to have instability, and that is the existence of real solutions,

$$
\left(\alpha_{3} N_{0}\right)^{2} \geqslant 4\left(D_{2} N_{0}^{2 / 3}+D_{3}\right)
$$

This expression gives us a threshold for $N_{0}$, and, consequently, a threshold for the flux through equation (17),

$$
\Gamma_{\mathrm{c}}=D_{1} N_{0}^{13 / 6}+D_{0} N_{0}
$$

This condition may not be sufficient. To have a sufficient condition for instability, there should be an integer $k$ between $k_{-}$and $k_{+}$. For a given $k$, the instability threshold condition, $\gamma=0$, reduces to a transcendent equation for $N_{0}$ :

$$
N_{0}=\frac{1+\left(D_{2} N_{0}^{2 / 3}+D_{3}\right)(k \pi)^{4}}{\alpha_{3}(k \pi)^{2}} .
$$

By applying equation (26) to different $k$-values, we can obtain the threshold for $\Gamma_{\mathrm{c}}$. We have done a scan in $D_{2}$ with $k$ going from 1 to 7 (figure 1), the rest of the parameters being

$$
D_{0}=10^{-3}, \quad D_{1}=10^{-2}, \quad D_{3}=10^{-6}, \quad \alpha_{3}=0.0175 .
$$

For high $D_{2}$-values, the solution of equation (26) can be approximated to

$$
N_{\mathrm{c}} \simeq \frac{(k \pi)^{6}}{\alpha_{3}^{3}} D_{2}^{3}, \quad \Gamma_{\mathrm{c}} \simeq \frac{(k \pi)^{13}}{\alpha_{3}^{13 / 2}} D_{1} D_{2}^{13 / 2} .
$$

This is the asymptotic behavior of the curves in figure 1 . 


\subsection{Stationary solutions near the fixed point}

We now look for stationary solutions with a given $k$-value, that is, with the poloidal flow shear at lowest order given by $\sigma(x)=\sigma_{10} \cos (k \pi x)$. From the stability properties of equation (8), the stationary solution is the fixed point solution (17) for values of the flux below $\Gamma_{\mathrm{c}}$. Since $\sigma^{2}=\sigma_{10}^{2}[1+\cos (2 k \pi x)] / 2$, we get from equations (7) and (15) that at first order, $E(x)=E_{\mathrm{s}}+E_{21} \cos (2 k \pi x)$, and $N(x)=N_{\mathrm{s}}+N_{21} \cos (2 k \pi x)$ for values of the flux above $\Gamma_{\mathrm{c}}$. By substituting these expansions in equations (8) and (15), we get at lowest order,

$$
\begin{aligned}
& 0=-1+\alpha_{3}(k \pi)^{2} N_{\mathrm{s}}^{-4 / 3} E_{\mathrm{s}}^{2}-(k \pi)^{4}\left(D_{2} N_{\mathrm{s}}^{-5 / 3} E_{\mathrm{s}}^{2}+D_{3}\right), \\
& \Gamma_{0}=N_{\mathrm{s}}\left(D_{1} E_{\mathrm{s}}+D_{0}\right) .
\end{aligned}
$$

By solving this system of two equations, we obtain $N_{\mathrm{s}}$ and $E_{\mathrm{s}}$.

By now substituting the expansions in equation (7), we get at lowest order,

$$
0=N_{\mathrm{s}}^{2 / 3} E_{\mathrm{s}}-N_{\mathrm{s}}^{-1 / 2} E_{\mathrm{s}}^{2}-\frac{\sigma_{10}^{2}}{2} N_{\mathrm{s}}^{-1 / 3} E_{\mathrm{s}} \Rightarrow \sigma_{10}^{2}=2\left(N_{\mathrm{s}}-N_{\mathrm{s}}^{-1 / 6} E_{\mathrm{s}}\right) .
$$

For the parameters we are using, $D_{0} \ll D_{1} E$, and $D_{3} \ll D_{2} N^{-5 / 3} E^{2}$, so from (16) and (17) we can approximate

$$
E_{0}=\left(\frac{\Gamma_{0}}{D_{1}}\right)^{7 / 13}, \quad N_{0}=\left(\frac{\Gamma_{0}}{D_{1}}\right)^{6 / 13}
$$

and equation (28) can be written

$$
\begin{aligned}
& N_{\mathrm{s}}=\frac{\Gamma_{0}}{D_{1} E_{\mathrm{s}}} \\
& 1=(k \pi)^{2}\left(\frac{\Gamma_{0}}{D_{1}}\right)^{-4 / 3} E_{\mathrm{s}}^{10 / 3}\left[\alpha_{3}-D_{2}(k \pi)^{2}\left(\frac{\Gamma_{0}}{D_{1}}\right)^{-1 / 3} E_{\mathrm{s}}^{1 / 3}\right] .
\end{aligned}
$$

Since the term between square brackets depends weakly on $E_{\mathrm{s}}$, we can approximate $E_{\mathrm{s}}$ in this term to the approximate value of $E_{0}$ for the critical flux, that is

$$
\left(\frac{\Gamma_{0}}{D_{1}}\right)^{-1 / 3} E_{\mathrm{s}}^{1 / 3} \simeq\left(\frac{\Gamma_{\mathrm{c}}}{D_{1}}\right)^{-2 / 13}
$$

so

$$
E_{\mathrm{s}}=E_{\mathrm{c}}\left(\frac{\Gamma_{0}}{\Gamma_{\mathrm{c}}}\right)^{2 / 5}
$$

where

$$
E_{\mathrm{c}}=(k \pi)^{-3 / 5}\left(\frac{\Gamma_{\mathrm{c}}}{D_{1}}\right)^{2 / 5}\left[\alpha_{3}-D_{2}(k \pi)^{2}\left(\frac{\Gamma_{\mathrm{c}}}{D_{1}}\right)^{-2 / 13}\right]^{-3 / 10}
$$

Then,

$$
N_{\mathrm{s}}=\frac{\Gamma_{\mathrm{c}}}{D_{1} E_{\mathrm{c}}}\left(\frac{\Gamma_{0}}{\Gamma_{\mathrm{c}}}\right)^{3 / 5}
$$

and from equation (30),

$$
\sigma_{10}^{2}=2\left[\frac{\Gamma_{\mathrm{c}}}{D_{1} E_{\mathrm{c}}}\left(\frac{\Gamma_{0}}{\Gamma_{\mathrm{c}}}\right)^{3 / 5}-E_{\mathrm{c}}^{7 / 6}\left(\frac{\Gamma_{\mathrm{c}}}{D_{1}}\right)^{-1 / 6}\left(\frac{\Gamma_{0}}{\Gamma_{\mathrm{c}}}\right)^{3 / 10}\right] .
$$




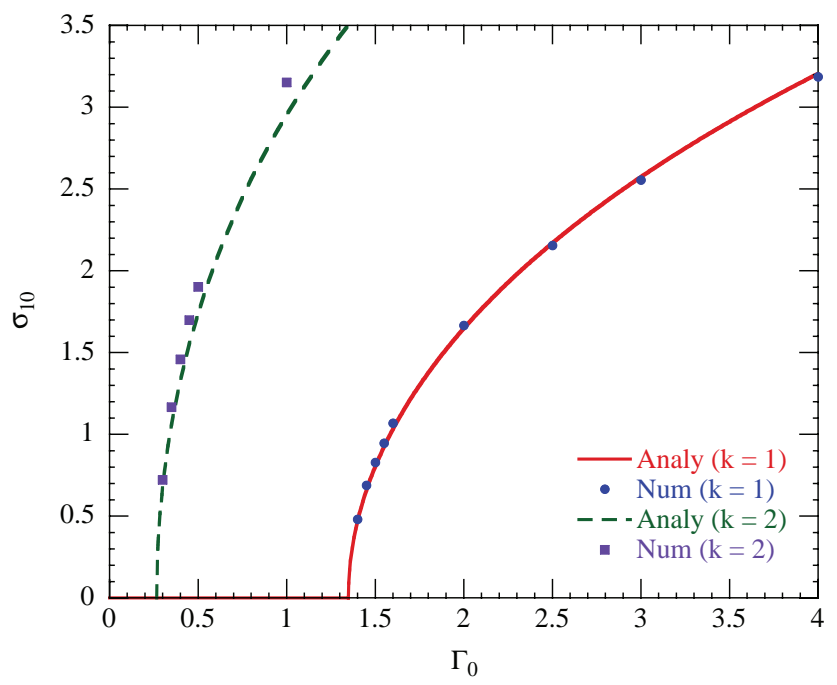

Figure 2. Comparison of analytical [equation (38), solid and broken lines] and numerical (dots and squares) results of the shear flow amplitude for stationary solutions when $D_{2}=1.5 \times 10^{-3}$ $(k=1)$ and $D_{2}=5 \times 10^{-4}(k=2)$.

Finally, from equations (7) and (15) we get, at second order,

$$
\begin{aligned}
& N_{21}=-\frac{N_{\mathrm{s}}}{E_{\mathrm{s}}} E_{21} \\
& E_{21}=\frac{\sigma_{10}^{2}}{2}\left[\frac{1}{3} \frac{N_{\mathrm{s}}}{E_{\mathrm{s}}}-\frac{5}{2} N_{\mathrm{s}}^{-1 / 6}-(2 k \pi)^{2} D_{1} N_{\mathrm{s}}^{1 / 3}\right]^{-1} .
\end{aligned}
$$

This gives a good approximation to the numerical results for $\Gamma_{0}$ close to the threshold. Since the experimental profiles of the edge shear flow and the fluctuation level in this region show few oscillations, we will focus our studies on the parameter space such as the most unstable modes are $k=1$ or 2 . In figure 2 we compare the analytical and numerical results for two different values of the parameter $D_{2}: D_{2}=1.5 \times 10^{-3}$ and $D_{2}=5 \times 10^{-4}$. For the first value of $D_{2}$, the most unstable mode corresponds to $k=1$, and for the second, to $k=2$. The flux thresholds for these parameters are 1.358 and 0.2703 , respectively. The analytical results are obtained from equation (38), and the numerical results are obtained by advancing numerically equations (7) to (9) until a stationary solution is reached. The comparisons of the analytical and numerical results for $E_{\mathrm{s}}$ and $E_{21}$ are also very good.

The profiles of $E$ and $\sigma$ for different values of the flux are shown in figure 3. They correspond to the scan in $\Gamma_{0}$ shown in figure 2 when $D_{2}=1.5 \times 10^{-3}$ and $k=1$. The comparison of the analytical (equations (31) and (35)) and numerical values of the fluctuation level for this scan is shown in figure 4. The fluctuation level always increases with the flux. However, the rate decreases when the flux is above the threshold from $\Gamma_{0}^{7 / 13}$ to $\Gamma_{0}^{2 / 5}$. This is consistent with the experimental results for these second-order transitions [10].

Let us consider, for example, the case $\Gamma_{0}=2$ of figure 3 . The approximate values of $E, \sigma$ and $N$ are $16.38,1.67$ and 12.15 , respectively. We can relate the dimensionless parameters with realistic physical magnitudes. For that, we assume a minor radius $a=0.5 \mathrm{~m}, L \sim 0.15 \mathrm{~m}$, $L_{\mathrm{p}}^{-1} \equiv-(1 / \mathcal{P}) \mathrm{d} \mathcal{P} / \mathrm{d} r \sim 4 \mathrm{~m}^{-1}, k_{\theta} \sim 20 \mathrm{~m}^{-1}$ and $\Lambda \sim 4$ [11]. Since, by definition, $N=\left(L / L_{\mathrm{p}}\right)\left(\mathcal{P} / \mathcal{P}_{0}\right), \mathcal{P} / \mathcal{P}_{0} \sim 20.25$. From equation (10), we get $W_{0} \sim 5.6 \times 10^{-3} \mathrm{~m}$, so 

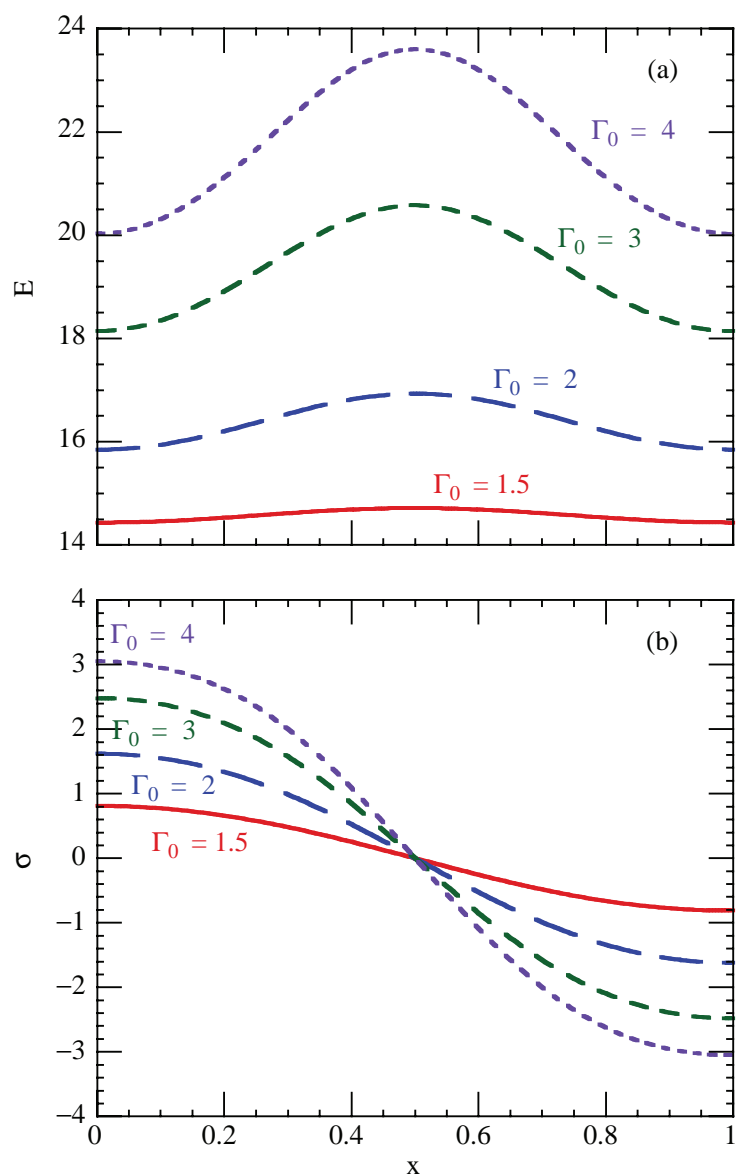

Figure 3. Numerical profiles of he fluctuation level $E(a)$ and the poloidal flow shear $\sigma(b)$ for some values of the flux of figure $2(k=1)$ above the threshold.

$W_{k} \sim 8.4 \times 10^{-3} \mathrm{~m}$. From equation (6), $\mathcal{E}_{0} \sim 9.24 \times 10^{-3}$, so $\mathcal{E} \sim 15 \%$. If we take a value of $\mu \sim 4 \times 10^{3} \mathrm{~s}^{-1}, \gamma=\mu N^{2 / 3} \sim 2 \times 10^{4} \mathrm{~s}^{-1}$, and from equation (6), we get $V^{\prime} \sim 6 \times 10^{4} \mathrm{~s}^{-1}$. These values are consistent with the experimental measurements in a medium size device.

In figure 2 the critical transition is shown as a series of stationary states. In order to get a dynamical transition we have done ramps in the flux. The evolution of the maximum of $\sigma$ is plotted in figures 5 and 6 for $D_{2}=1.5 \times 10^{-3}$ and $D_{2}=5 \times 10^{-4}$, respectively. It is compared with the maximum of $\sigma$ for a series of stationary solutions. The transition is less abrupt than in the stationary case, and the flux threshold increases with the flux rate. After the transition, there is a phase of rapid growth of the poloidal flow shear, and the stationary value of $\sigma$ is eventually reached when the energy flux is far above the threshold. The evolution of the minimum values of $\sigma$ and the minimum and maximum values of $E$ for the ramp with $D_{2}=1.5 \times 10^{-3}$ and $\mathrm{d} \Gamma_{0} / \mathrm{d} t=0.1$ is shown in figure 7 . There is a delay in the decrease in fluctuations with respect to the formation of the shear layer, as happens in the experiment. At the end of the ramp, when the energy flux is far above the threshold, the fluctuation level $E$ increases at a slower rate than below the threshold, as in the series of stationary solutions. 


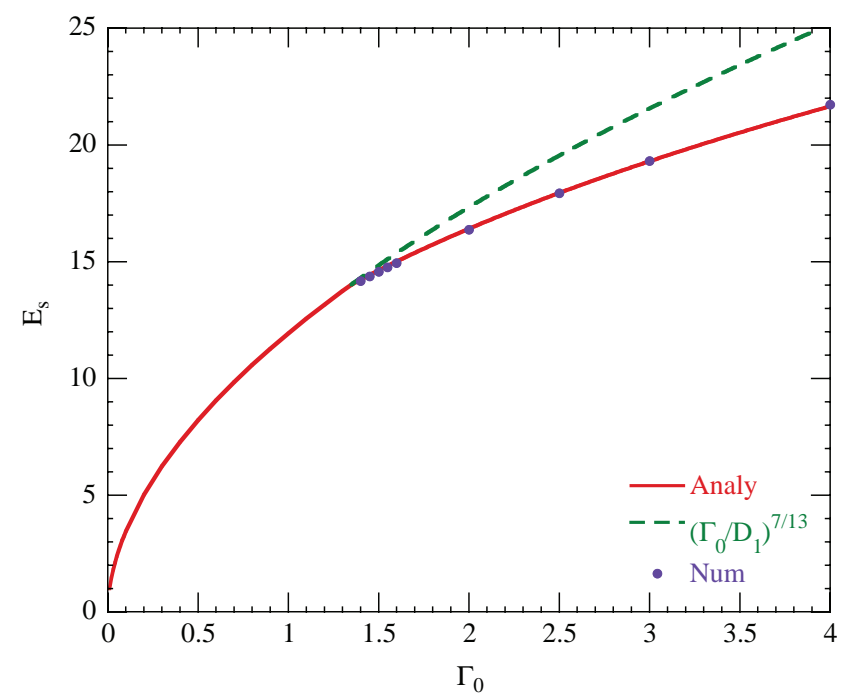

Figure 4. Comparison of analytical [equations (31) and (35), solid line] and numerical (dots) results of the fluctuation amplitude $E_{\mathrm{s}}$. The fixed point solution $\left(\Gamma_{0} / D_{1}\right)^{7 / 13}$ is extended for values of $\Gamma_{0}$ above $\Gamma_{\mathrm{c}}$ (broken line).

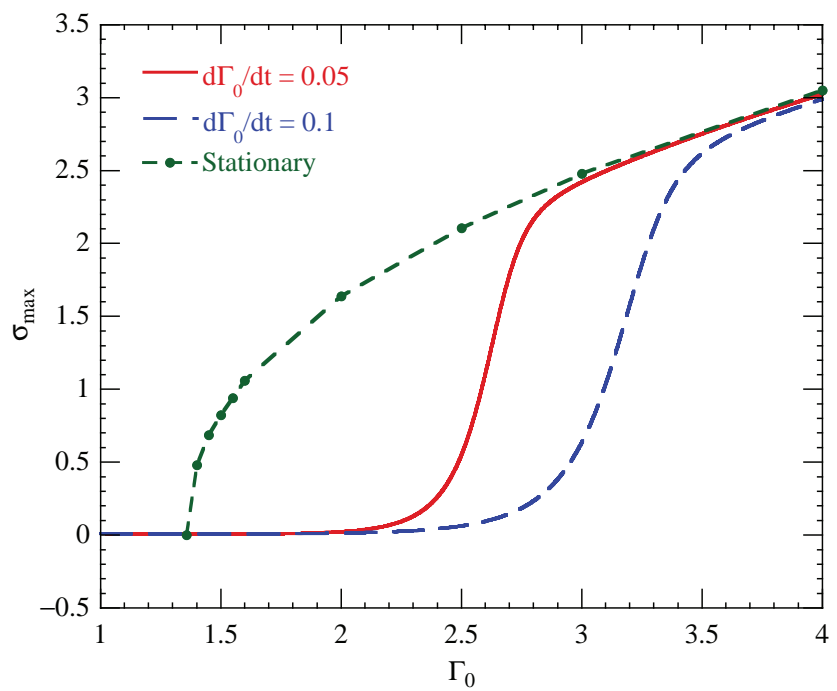

Figure 5. Dynamic evolution of maximum $\sigma$ when $D_{2}=1.5 \times 10^{-3}$ for two different flux rates. The amplitude of $\sigma$ for a series of stationary solutions (dots) is shown for comparison.

\section{Effect of electrode biasing}

To study the effect of electrode biasing, we add an external torque term $\tau=\tau_{0} \cos (k \pi x)$, to the r.h.s. of equation (8), so the equation for the shear flow is now

$$
\frac{\partial \sigma}{\partial t}=-\sigma-\alpha_{3} \frac{\partial^{2}}{\partial x^{2}}\left(N^{-4 / 3} E^{2} \sigma\right)-\frac{\partial^{2}}{\partial x^{2}}\left[\left(D_{2} N^{-5 / 3} E^{2}+D_{3}\right) \frac{\partial^{2} \sigma}{\partial x^{2}}\right]+\tau_{0} \cos (k \pi x) .
$$




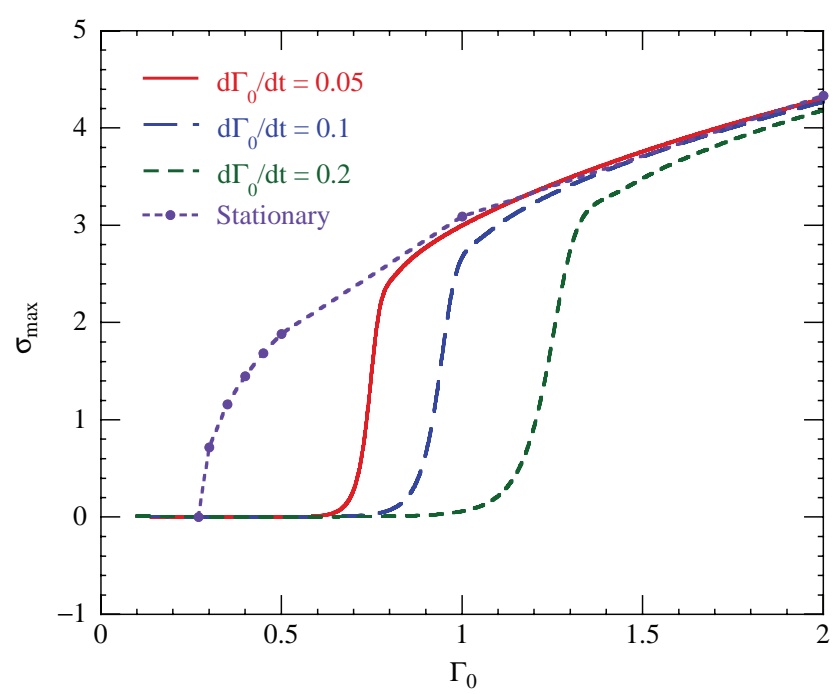

Figure 6. Dynamic evolution of maximum $\sigma$ when $D_{2}=5 \times 10^{-4}$ for three different flux rates. The amplitude of $\sigma$ for a series of stationary solutions (dots) is shown for comparison.

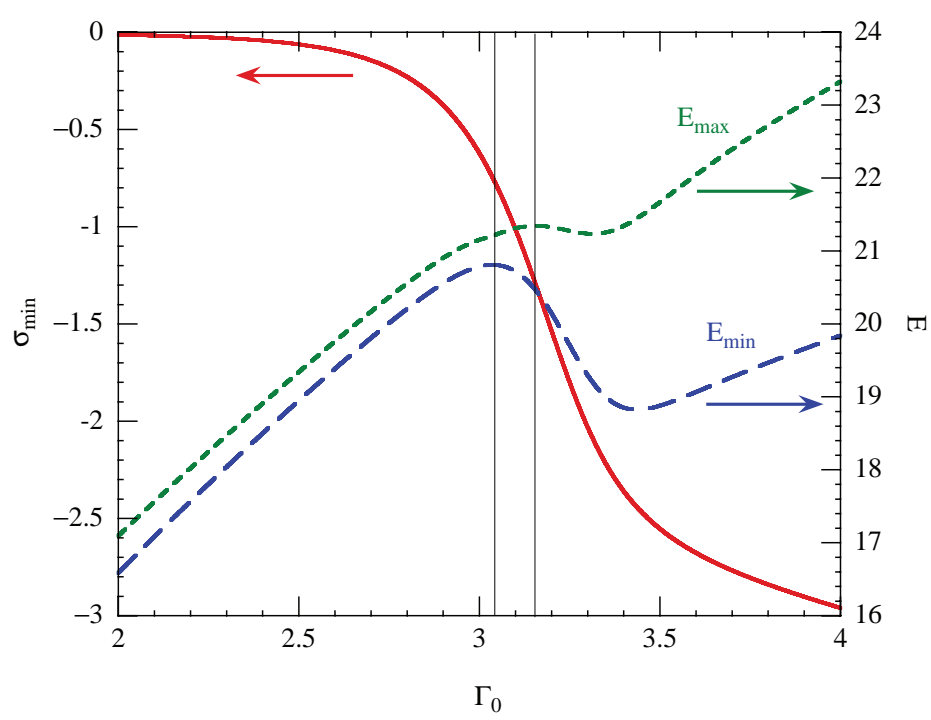

Figure 7. Dynamic evolution of minimum $\sigma$ and minimum and maximum $E$ for the flux rate of 0.1 of figure 5 . The vertical lines indicate the points where the minimum and maximum $E$ reach a maximum.

For low values of $\tau_{0}$, we can use the same expansion as before assuming $\sigma$ of order $\delta$. However, for moderate values of $\tau_{0}$, the third term of the r.h.s. of equation (7) cannot be assumed of higher order than the first two terms. So, in general, we will write

$$
\begin{aligned}
& E=E_{\mathrm{s}}+\tilde{E} \\
& N=N_{\mathrm{s}}+\tilde{N} \\
& \sigma=\sigma_{1} \cos (k \pi x)+\tilde{\sigma}
\end{aligned}
$$




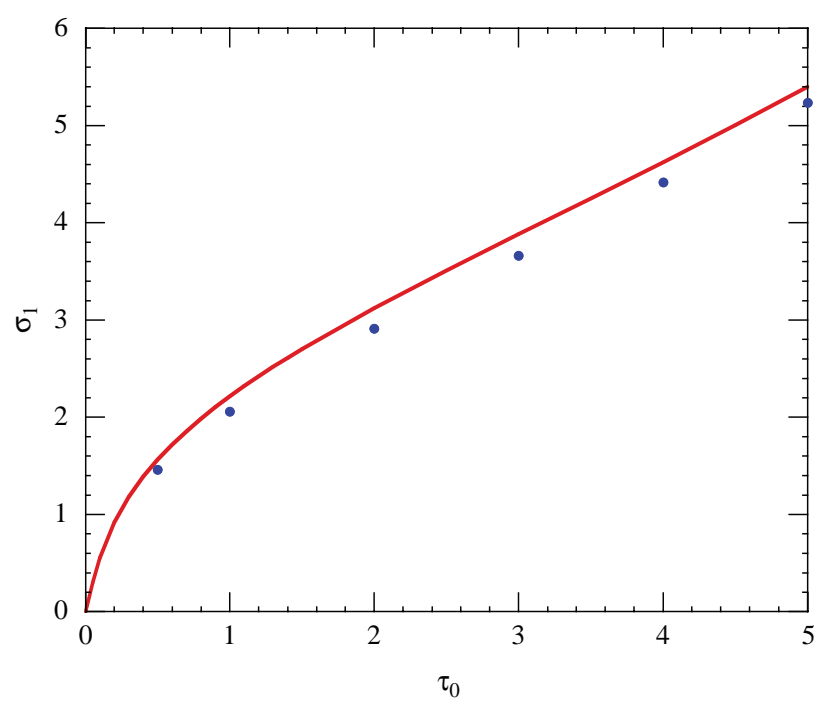

Figure 8. Comparison of the values of the $k=1$ amplitude of $\sigma$ as a function of the external torque obtained by solving the system of equations (43) (solid line) and from the numerical stationary solutions (dots). The energy flux for this scan is 1 , below the critical value.

and we will assume that

$\tilde{E}=E_{2} \cos (2 k \pi x)+\cdots, \quad \tilde{N}=N_{2} \cos (2 k \pi x)+\cdots, \quad \tilde{\sigma}=\sigma_{3} \cos (3 k \pi x)+\cdots$,

where

$$
\left|E_{2}\right| \ll E_{\mathrm{s}}, \quad\left|N_{2}\right| \ll N_{\mathrm{s}}, \quad\left|\sigma_{3}\right| \ll\left|\sigma_{1}\right| .
$$

From equations (7), (41) and (15), we get, at lowest order,

$$
\begin{aligned}
& N_{\mathrm{s}}-N_{\mathrm{s}}^{-1 / 6} E_{\mathrm{s}}-\frac{1}{2} \sigma_{1}^{2}=0 \\
& {\left[-1+\alpha_{3}(k \pi)^{2} N_{\mathrm{s}}^{-4 / 3} E_{\mathrm{s}}^{2}-(k \pi)^{4}\left(D_{2} N_{\mathrm{s}}^{-5 / 3} E_{\mathrm{s}}^{2}+D_{3}\right)\right] \sigma_{1}+\tau_{0}=0} \\
& N_{\mathrm{s}}\left(D_{1} E_{\mathrm{s}}+D_{0}\right)=\Gamma_{0} .
\end{aligned}
$$

By solving this system of equations we can obtain $E_{\mathrm{s}}, \sigma_{1}$ and $N_{\mathrm{s}}$. The solution for $\Gamma_{0}=1$, $D_{2}=1.5 \times 10^{-3}$ and $k=1$ is shown in figure 8 (solid line). Also in figure 8 we plot the numerical values for the stationary states (dots). To obtain the numerical values of $\sigma_{1}$, we have fitted the $\sigma$-profiles to $\sigma_{1} \cos (k \pi x)+\sigma_{3} \cos (3 k \pi x)$.

In the rest of the calculations of this paper, we apply the electrode biasing during a time $t=10$, and then we switch off the biasing to analyze the decay of the shear flow. From equation (41) it is clear that the decay rate is not necessarily going to agree with neoclassical damping (first term of the r.h.s.). Turbulent damping mechanisms through Reynolds stress (second and third terms) are likely to modify its value. The evolution of the integral of $\sigma^{2}$ for different values of $\tau_{0}$ when $\Gamma_{0}=1$ is shown in figure 9. For this scan, $D_{2}=1.5 \times 10^{-3}$, and $k=1$, so we are below the threshold flux in the absence of biasing (subcritical regime). In most of the cases the shear flow has two decay scales and the change between them is more pronounced as $\tau_{0}$ increases. The square root of the integral of $\sigma^{2}$ decays like e ${ }^{\gamma_{1} t}$ just after switching off the biasing (first decay region), and like $\mathrm{e}^{\gamma_{2} t}$ at larger times (second decay region). The first decay rate is easily understood from equation (8). As we switch off the biasing, the 


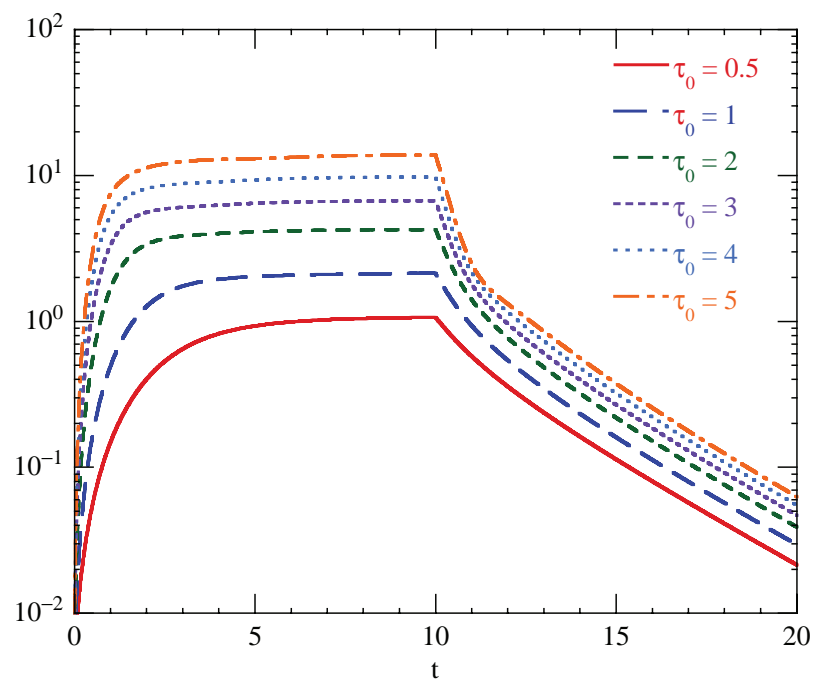

Figure 9. Evolution of the integral of $\sigma^{2}$ for the stationary solutions of figure 8. At $t=10$, after reaching the stationary solution, the external torque is suppressed.

instantaneous exponential decay rate will be

$$
\gamma_{1}=-1+\alpha_{3}(k \pi)^{2} N_{\mathrm{s}}^{-4 / 3} E_{\mathrm{s}}^{2}-(k \pi)^{4}\left(D_{2} N_{\mathrm{s}}^{-5 / 3} E_{\mathrm{s}}^{2}+D_{3}\right),
$$

or equivalently,

$$
\gamma_{1}=-\frac{\tau_{0}}{\sigma_{1}}
$$

where $\sigma_{1}$ corresponds to the stationary state with external torque $\tau_{0}$. The second exponential decay rate is very similar for all the cases, with $\gamma_{2}$-values between -0.16 and -0.18 .

The evolution of the integral of $\sigma^{2}$ when $\Gamma_{0}=1.35$ is shown in figure 10 . This value of $\Gamma_{0}$ is very close to the critical value, $\Gamma_{\mathrm{c}}=1.35794$. For this scan, the evolution of the integral of $\sigma^{2}$ when we switch off the biasing is no longer exponential. Instead, the decay is algebraic, and can be fitted to $C /(1+\Delta t / T)$, where $C$ is a constant (value of the integral when we switch off the biasing), and $\Delta t=t-10$ is the elapsed time after suppression of the external torque. The fit begins to fail for values of $\tau_{0}$ around 1 . For very high values of $\tau_{0}$, there is an oscillatory behavior after switching off the biasing. The parameter $T$ has a power-law dependence on $\tau_{0}$, as can be seen in figure 11 .

We have also tried scans in $\tau_{0}$ with values of $\Gamma_{0}$ above the threshold (supercritical regime), in particular $\Gamma_{0}=1.37$ and 1.5. For these cases, the evolution of the integral of $\sigma^{2}$ when we suppress the external torque can be fitted to $C /(1+\Delta t / T)+C_{0}$, where $C_{0}$ is a constant with a value close to the integral of $\sigma^{2}$ for the stationary state in the absence of biasing.

Finally, the results of the evolution of the integral of $\sigma^{2}$ for scans in $\Gamma_{0}$ when $D_{2}=$ $1.5 \times 10^{-3}$ and $D_{2}=5 \times 10^{-4}$ are shown in figures 12 and 13 , respectively. In the $y$-axis, we represent the integral of $\sigma^{2}$ when we switch off the biasing minus the value for the stationary state in absence of biasing. For each case, the slower decay corresponds to the flux value closer to $\Gamma_{\mathrm{c}}, \Gamma_{0}=1.35$ for $D_{2}=1.5 \times 10^{-3}$ and $\Gamma_{0}=0.27$ for $D_{2}=5 \times 10^{-4}$. The decay time tends to infinity as $\Gamma_{0}$ tends to the critical value $\Gamma_{\mathrm{c}}$. Even though the decay is algebraic when the flux is close to $\Gamma_{\mathrm{c}}$, we have fitted the data of the evolution of the squared root of the integral of $\sigma^{2}$ for each $\Gamma_{0}$-value to $C \exp (-t / T)+C_{0}$ when $D_{2}=1.5 \times 10^{-3}$, as is done in biasing experiments in TJ-II [13]. The results for the decay time $T$ are shown in figure 14. The slower 


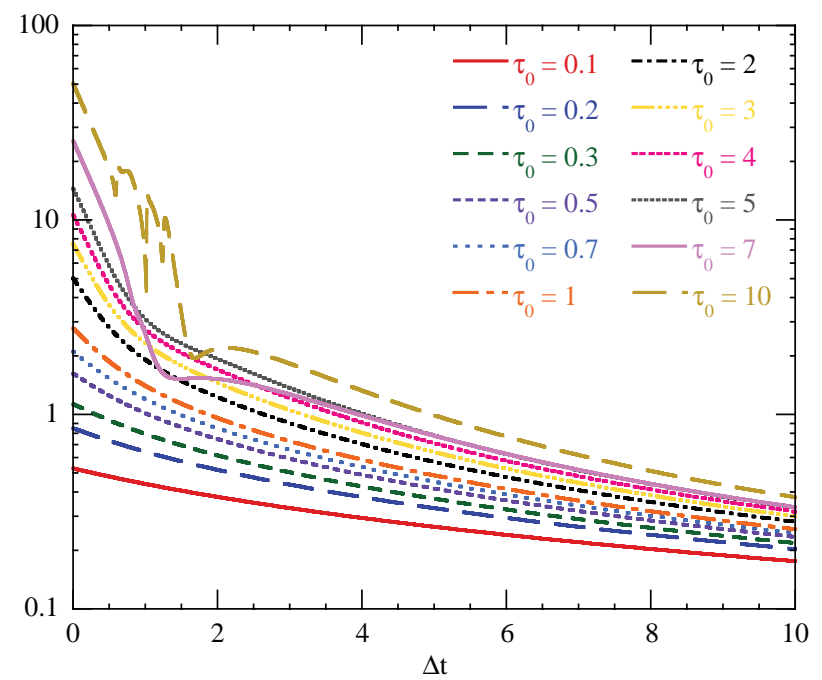

Figure 10. Evolution of the integral of $\sigma^{2}$ after switching off the biasing at $\Delta t=0$. The value of the energy flux is 1.35 , just below the critical value $\Gamma_{\mathrm{c}}=1.35794$.

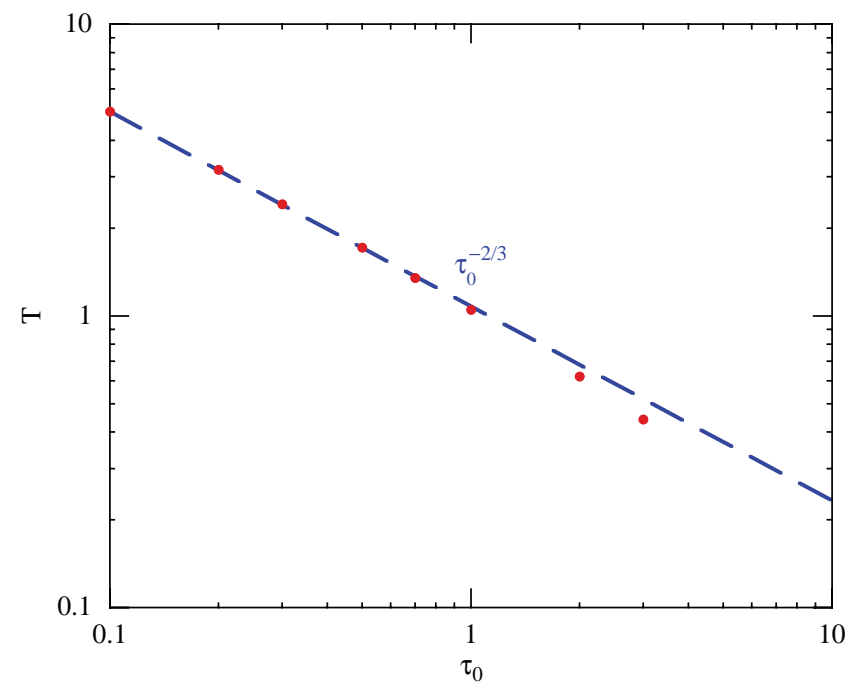

Figure 11. Decay time $T$ as a function of $\tau_{0}$ for the cases of figure 10. The time dependence of the integral of $\sigma^{2}$ after switching off the biasing is fitted to $C /(1+\Delta t / T)$, where $C$ is a constant.

decays correspond to flux values closer to $\Gamma_{\mathrm{c}}$. The damping is close to the viscous damping (one in our units) only when the flux is far above the threshold. The results are similar when the value of the external torque is changed.

\section{Conclusions}

Motivated by the experimental possibility of high spatial resolution diagnostics, the radial structure of high confinement modes is investigated. Hence, a simplified model describing the 


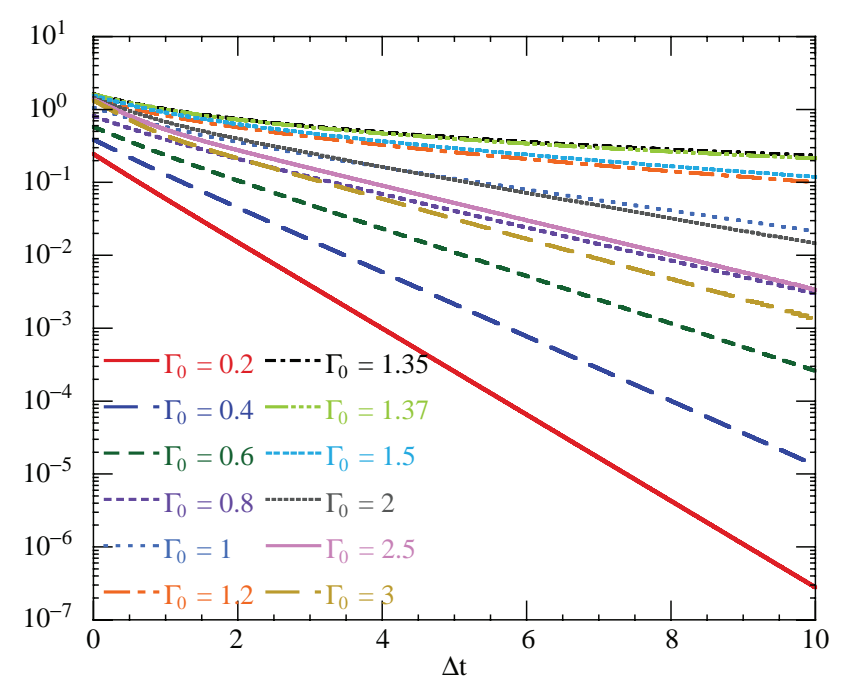

Figure 12. Evolution of the integral of $\sigma^{2}$ after switching off the biasing for various values of the energy flux when $D_{2}=1.5 \times 10^{-3}$. The value of the integral of $\sigma^{2}$ for the stationary solutions in the absence of biasing has been subtracted in the cases with flux above the threshold. The amplitude of the external torque is $\tau_{0}=0.5$.

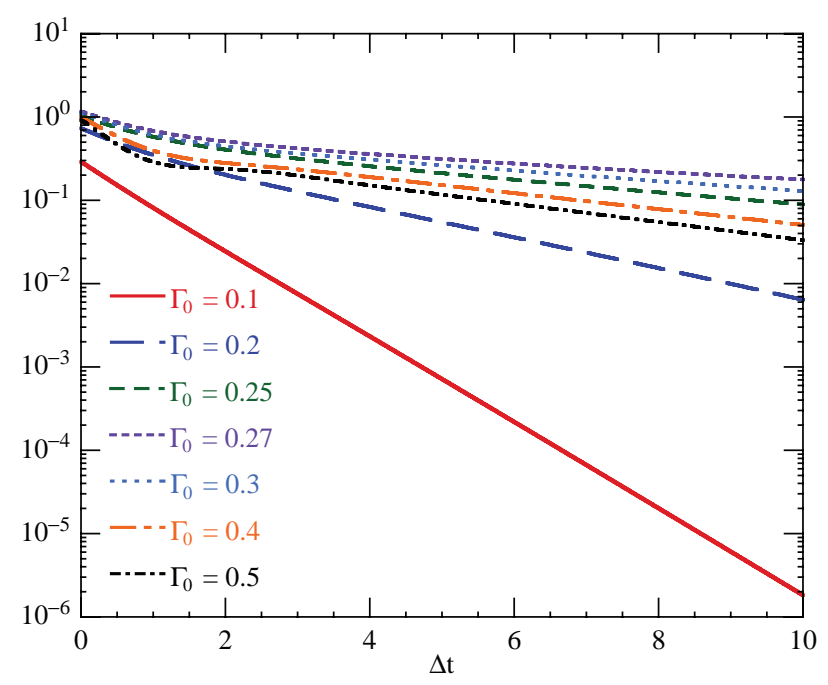

Figure 13. The same as figure 12 when $D_{2}=5 \times 10^{-4}$.

self-consistent interaction of fluctuations, shear flow and pressure gradient has been developed. In this model we include the dependence of the coefficients on the pressure gradient, and an expression for the Reynolds stress term has been derived. Analytical and numerical solutions show a second-order transition as we increase the energy flux. For values of the flux higher than a critical value, a shear flow is spontaneously developed and the fluctuation level increases at a slower rate.

The effect of electrode biasing has been included in the model through an external torque. We apply the electrode biasing during a time and then we switch off the biasing to analyze the 


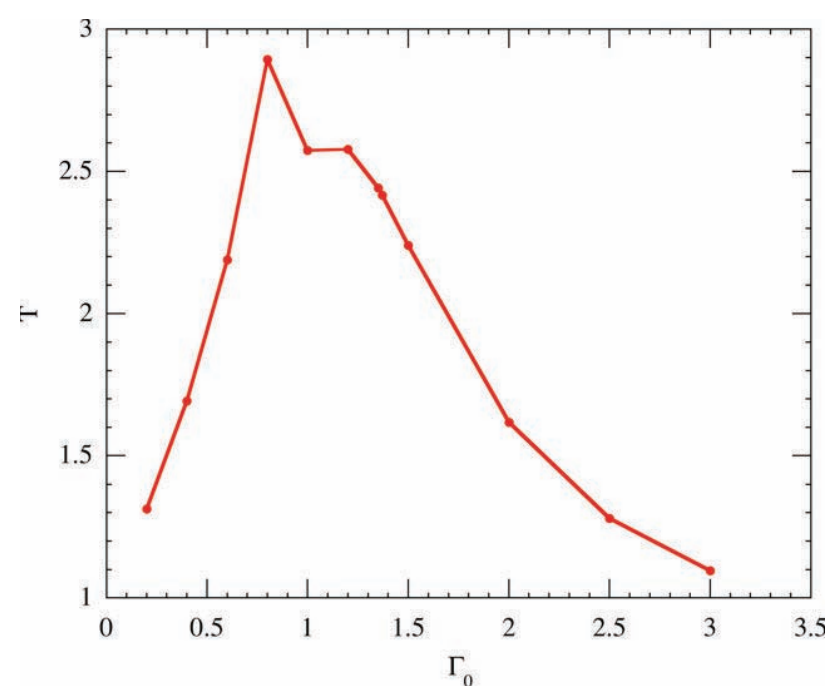

Figure 14. Decay time $T$ as a function of the energy flux for the cases of figure 12 . The time dependence of the integral of $\sigma^{2}$ after switching off the biasing is fitted to $C \exp (-t / T)+C_{0}$, where $C$ and $C_{0}$ are constants. The slower decay is close to the critical flux.

decay of the shear flow. The decay is algebraic when the flux is close to the critical value, and the decay time tends to infinity as the flux tends to the critical value. Results are consistent with the experiment.

\section{Acknowledgments}

The authors would like to thank I Calvo for helpful discussions. This research was sponsored in part by the DGI (Dirección General de Investigación) of Spain under project No ENE200615244-C03-01 and by CM-UC3M (Comunidad de Madrid - Universidad Carlos III) Project No CCG07-UC3M/ESP-3407.

\section{Appendix A. Reynolds stress calculation}

Appendix A.1. Single mode case

In a single mode calculation the fluctuating component of the velocity stream function is

$$
\tilde{\phi}=\mathrm{e}^{\left(-\mathrm{i} \omega t+\mathrm{i} k_{y} \bar{y}+\mathrm{i} k_{z} \bar{z}\right)} \phi(\bar{x}),
$$

where $\bar{x}$ is the radial coordinate with the origin at the rational surface, $\bar{y}=r \theta$, and $\bar{z}=R_{0} \varphi$.

The Reynolds stress in terms of the stream function is

$$
\left\langle\widetilde{V}_{r} \widetilde{V}_{\theta}\right\rangle=-\frac{k_{y}}{2}\left(\phi_{R} \frac{\mathrm{d} \phi_{I}}{\mathrm{~d} \bar{x}}-\phi_{I} \frac{\mathrm{d} \phi_{R}}{\mathrm{~d} \bar{x}}\right) .
$$

We use the relation $\xi / W=\omega_{\mathrm{s}} / \gamma$, where $\xi$ is the phase, $W$ is the radial width, $\gamma$ is the growth rate and $\omega_{\mathrm{s}}$ is the shearing rate [14]. Using the explicit expression for this term we arrive at the following expression:

$$
\left\langle\widetilde{V}_{r} \widetilde{V}_{\theta}\right\rangle=\left.\frac{1}{2} \frac{1}{\gamma}\left|k_{y} \phi(\bar{x})\right|^{2} \frac{\mathrm{d} V_{\theta}}{\mathrm{d} \bar{x}}\right|_{\bar{x}=0} .
$$


Assuming purely convective plasma, this expression can be rewritten in terms of the fluctuation density as

$$
\left\langle\widetilde{V}_{r} \widetilde{V}_{\theta}\right\rangle=\left.\frac{1}{2} \gamma \mathcal{E}^{2}\left(\frac{1}{\mathcal{P}} \frac{\mathrm{d} \mathcal{P}}{\mathrm{d} \bar{x}}\right)^{-2} \frac{\mathrm{d} V_{\theta}}{\mathrm{d} \bar{x}}\right|_{\bar{x}=0}
$$

\section{Appendix A.2. Multiple modes}

We assume that we have a uniform distribution of modes. Each mode is localized at a singular surface $r_{i}$, where $i$ is the index of the surface. Therefore, the Reynolds stress is the sum of the contributions from all these modes in the following way.

$$
\left\langle\widetilde{V}_{r} \widetilde{V}_{\theta}\right\rangle=\left.\frac{1}{2} \sum_{i=1} \frac{1}{\gamma_{i}}\left|k_{y} \phi\left(r-r_{i}\right)\right|^{2} \frac{\mathrm{d} V_{\theta}}{\mathrm{d} r}\right|_{r=r_{i}}
$$

If we approximate the summation by a continuous integral over $r_{i}$ and we change integration variables to $y=r-r_{i}$, we have

$$
\begin{aligned}
\left\langle\widetilde{V}_{r} \widetilde{V}_{\theta}\right\rangle & \approx \frac{1}{2 \gamma} \int_{-\infty}^{\infty}\left|k_{y} \phi(y)\right|^{2} \frac{\mathrm{d} V_{\theta}(r+y)}{\mathrm{d} r} \\
& \approx \frac{1}{2 \gamma}\left\{\frac{\mathrm{d} V_{\theta}}{\mathrm{d} r} \int_{-\infty}^{\infty} \mathrm{d} y\left|k_{y} \phi(y)\right|^{2}+\frac{\mathrm{d}^{3} V_{\theta}}{\mathrm{d} r^{3}} \int_{-\infty}^{\infty} \mathrm{d} y y^{2}\left|k_{y} \phi(y)\right|^{2}+\cdots\right\} .
\end{aligned}
$$

We can write this expression in terms of the density fluctuations. The integration can be interpreted as integrating over the fast varying scale.

$$
\left\langle\widetilde{V}_{r} \widetilde{V}_{\theta}\right\rangle \approx \frac{1}{2}\left\{\gamma\left(\frac{1}{\mathcal{P}} \frac{\mathrm{d} \mathcal{P}}{\mathrm{d} r}\right)^{-2} \frac{\mathrm{d} V_{\theta}}{\mathrm{d} r} \mathcal{E}(r)^{2}+\gamma W^{2}\left(\frac{1}{\mathcal{P}} \frac{\mathrm{d} \mathcal{P}}{\mathrm{d} r}\right)^{-2} \frac{\mathrm{d}^{3} V_{\theta}}{\mathrm{d} r^{3}} \mathcal{E}(r)^{2}\right\} .
$$

This form of the Reynolds stress conserves momentum. Finally we obtain equation (8).

\section{References}

[1] Burrell K H 1997 Phys. Plasmas 41499

[2] Terry P W 2000 Rev. Mod. Phys. 72109

[3] Alejaldre C et al 1990 Fusion Technol. 17131

[4] Hidalgo C, Pedrosa M A, Garcia L and Ware A 2004 Phys.Rev. E 70067402

[5] Pedrosa M A et al 2005 Plasma Phys. Control. Fusion 47777

[6] Gonçalves B, Hidalgo C, Pedrosa M A, Orozco R O, Sánchez E and Silva C 2006 Phys. Rev. Lett. 96145001

[7] Diamond P H, Liang Y-M, Carreras B A and Terry P W 1994 Phys. Rev. Lett. 722565

[8] Wagner F et al 1982 Phys. Rev. Lett. 491408

[9] del-Castillo-Negrete D and Carreras B A 2002 Phys.Plasmas 9118

[10] Carreras B A, Garcia L, Pedrosa M A and Hidalgo C 2006 Phys. Plasmas 13122509

[11] Carreras B A, Garcia L and Diamond P H 1987 Phys. Fluids 301388

[12] Diamond P H, Itoh S-I, Itoh K and Hahm T S 2005 Plasma Phys. Control. Fusion 47 R35

[13] Pedrosa M A et al 2007 Plasma Phys. Control. Fusion 49 B303

[14] Carreras B A, Lynch V E, Garcia L and Diamond P H 1993 Phys. Fluids B 51491 